\title{
The optical gravitational lensing experiment. Variable stars in globular clusters. I. Fields 5139A-C in $\omega$ Centauri $^{\star}$
}

\author{
J. Kaluzny ${ }^{1}$, M. Kubiak ${ }^{1}$, M. Szymański ${ }^{1}$, A. Udalski ${ }^{1}$, W. Krzemiński ${ }^{2}$ and M. Mateo ${ }^{3}$ \\ 1 Warsaw University Observatory, Al. Ujazdowskie 4, 00-478 Warsaw, Poland \\ e-mail: (jka,mk,msz,udalski)@sirius.astrouw.edu.pl \\ 2 Carnegie Observatories, Las Campanas Observatory, Casilla 601, La Serena, Chile \\ e-mail: wojtek@roses.ctio.noao.edu \\ 3 Department of Astronomy, University of Michigan, 821 Dennison Bldg., Ann Arbor, MI 48109-1090, U.S.A. \\ e-mail: mateo@astro.lsa.umich.edu
}

Received January 3; accepted April 15, 1996

\begin{abstract}
Three fields covering the central part of the globular cluster $\omega$ Cen were surveyed in a search for variable stars. We present $V$-band light curves for 39 periodic variables: 24 SX Phe stars, 7 contact binaries, 5 detached or semi-detached binaries, and 3 likely spotted variables (FK Com or RS CVn type stars). Only 2 of these variables were previously known. All SX Phe stars and all contact binaries from our sample belong to blue stragglers. Observed properties of these stars are consistent with their cluster membership. Of particular interest is detection of two well detached binaries with periods $P=1.50$ day and $P=2.47$ day. Further study of these two binaries can provide direct information about properties of turnoff stars in $\omega$ Cen. An incomplete light curve of a Mira variable known as $V 2$ was obtained. We present $V$ vs. $V-I$ color-magnitude diagrams for the monitored part of the cluster.
\end{abstract}

Key words: globular clusters: individual: NGC 5139 — star: variables: other — blue stragglers — binaries: eclipsing - HR diagram

\section{Introduction}

The Optical Gravitational Lensing Experiment (OGLE) is a long term project with the main goal of searching for dark matter in our Galaxy by identifying microlensing events toward the galactic bulge (Udalski et al. 1992, 1994a). At times the Bulge is unobservable we conduct other long-term photometric programs. A complete list of side-projects attempted by the OGLE team can be found in Paczyński et al. (1995). In particular, we monitored globular clusters NGC 104 (=47 Tuc) and NGC $5139(=\omega$ Cen $)$ in a search for variable stars of various types. Of primary interest was detection of detached eclipsing binaries. Such binaries can potentially provide a vital information about masses of stars in globular clusters. We expected also to detect some contact binaries and pulsating variables of SX Phe type.

It has been known for a long time that binaries play an important role in the dynamical evolution of globular

Send offprint requests to: J. Kaluzny

${ }^{\star}$ Based on observations collected at the Las Campanas Observatory of the Carnegie Institution of Washington clusters. Different types of binary stars are known to occur in globular clusters (see the detailed review by Hut et al. 1992). Surprisingly very few globular clusters were searched thoroughly for eclipsing binaries. So far positive results were published for NGC 5466 (Mateo et al. 1990), NGC 4371 (Kaluzny \& Krzemiński 1993) and M71 (Yan \& Mateo 1995; Hodder et al. 1992). Niss et al. (1978) discovered several faint variables in $\omega$ Cen through visual inspection of photographic plates. One of these star, known as NJL-5, turned out to be an eclipsing binary. That was in fact the first variable of this type discovered in any globular cluster. The complete light curve of NJL-5 was published by Jensen \& Jorgensen (1985). Recently Gilliland et al. (1995) reported discovery of two eclipsing binaries in the core of 47 Tuc in the data obtained with the HST.

In this paper we report discovery of 11 eclipsing binaries and 23 SX Phe stars in the field of $\omega$ Cen. In addition we obtained light curves for 3 previously known variables - one eclipsing binary, one SX Phe star and one Mira-type star. Photometry of RR Lyr variables in this cluster as 
Table 1. Equatorial coordinates for centers of $\omega$ Cen fields monitored for variables by OGLE in 1993 and 1994 seasons

\begin{tabular}{lll}
\hline Field & $\alpha_{2000}$ & $\delta_{2000}$ \\
\hline 5139A & $13: 26: 38.6$ & $-47: 15: 40$ \\
5139B & $13: 26: 43.8$ & $-47: 28: 52$ \\
5139BC & $13: 26: 42.5$ & $-47: 29: 27$ \\
5139C & 132650.8 & -474214 \\
\hline
\end{tabular}

Table 2. Number of frames reduced for the $\omega$ Cen fields

\begin{tabular}{lll}
\hline Field & Filter $V$ & Filter $I$ \\
\hline 5139A & 238 & 1 \\
5139B & 152 & 1 \\
5139BC & 227 & 67 \\
5139C & 292 & 35 \\
\hline
\end{tabular}

Table 3. Basic statistical data about stars from fields 5139A and $5139 \mathrm{C}$ examined for variability using an $\chi^{2}$ test. The data are given for bins 0.5 mag wide. Columns 2 and 5 give median value of rms for a given bin. Columns 3 and 6 give number of stars examined for variability while Cols. 4 and 7 give number of suspected variables selected using the $\chi^{2}$ test

\begin{tabular}{ccrrcrr}
\hline & $\mathrm{A}$ & & \multicolumn{2}{c}{$\mathrm{C}$} \\
$V$ & $<\mathrm{rms}>$ & $N$ & $\mathrm{VAR}$ & $\langle\mathrm{rms}\rangle$ & $N$ & VAR \\
\hline 14.5 & 0.011 & 100 & 18 & 0.013 & 86 & 5 \\
15.0 & 0.009 & 168 & 9 & 0.013 & 161 & 2 \\
15.5 & 0.009 & 153 & 6 & 0.013 & 139 & 3 \\
16.0 & 0.011 & 202 & 14 & 0.013 & 132 & 2 \\
16.5 & 0.013 & 276 & 6 & 0.015 & 244 & 4 \\
17.0 & 0.017 & 401 & 18 & 0.017 & 339 & 25 \\
17.5 & 0.022 & 877 & 61 & 0.022 & 717 & 41 \\
18.0 & 0.027 & 1946 & 141 & 0.028 & 1633 & 137 \\
18.5 & 0.038 & 3255 & 338 & 0.039 & 2813 & 263 \\
19.0 & 0.055 & 4500 & 501 & 0.056 & 4094 & 500 \\
\hline
\end{tabular}

Table 4. Same as Table 3 but for inner parts of fields 5139B and 5139BC

\begin{tabular}{ccrrcrr}
\hline $\begin{array}{c}\text { noalign } \\
V\end{array}$ & $\begin{array}{c}\mathrm{B} \\
<\mathrm{rms}>\end{array}$ & $N$ & $\mathrm{VAR}$ & $\begin{array}{c}\text { BC } \\
\text { rms }>\end{array}$ & $N$ & VAR \\
\hline 14.5 & 0.012 & 86 & 6 & 0.027 & 206 & 32 \\
15.0 & 0.012 & 105 & 2 & 0.025 & 359 & 35 \\
15.5 & 0.014 & 105 & 2 & 0.026 & 327 & 31 \\
16.0 & 0.019 & 118 & 2 & 0.031 & 392 & 62 \\
16.5 & 0.028 & 194 & 14 & 0.041 & 577 & 134 \\
17.0 & 0.037 & 285 & 25 & 0.059 & 1199 & 403 \\
17.5 & 0.046 & 558 & 51 & 0.077 & 2555 & 1039 \\
18.0 & 0.064 & 975 & 143 & & & \\
\hline
\end{tabular}

Table 5. Same as Table 3 but for outer parts of fields 5139B and 5139BC

\begin{tabular}{ccrrcrr}
\hline & $\mathrm{B}$ & & & $\mathrm{BC}$ & \\
$V$ & $\langle\mathrm{rms}\rangle$ & $N$ & VAR & $\langle$ rms $>$ & $N$ & VAR \\
\hline 14.5 & 0.009 & 84 & 12 & 0.017 & 87 & 12 \\
15.0 & 0.010 & 123 & 4 & 0.014 & 128 & 6 \\
15.5 & 0.011 & 114 & 2 & 0.013 & 118 & 2 \\
16.0 & 0.014 & 144 & 4 & 0.015 & 164 & 2 \\
16.5 & 0.018 & 236 & 8 & 0.018 & 241 & 6 \\
17.0 & 0.024 & 334 & 8 & 0.024 & 328 & 10 \\
17.5 & 0.032 & 874 & 36 & 0.029 & 847 & 53 \\
18.0 & 0.046 & 2002 & 166 & 0.038 & 1825 & 208 \\
18.5 & 0.065 & 3282 & 332 & 0.053 & 3042 & 473 \\
\hline
\end{tabular}

Table 6. Number of stars selected as potential variables by scanning light curves with a filter designed to pick-up eclipse-like events. Columns 2-5 give results for fields 5139A, $5139 \mathrm{C}$ and for the outer parts of fields 5139B and 5139BC

\begin{tabular}{crrrr}
\hline & $\mathrm{A}$ & $\mathrm{C}$ & $\mathrm{B}$ & $\mathrm{BC}$ \\
$V$ & $N_{\mathrm{VAR}}$ & $N_{\mathrm{VAR}}$ & $N_{\mathrm{VAR}}$ & $N_{\mathrm{VAR}}$ \\
\hline 14.5 & 15 & 5 & 9 & 10 \\
15.0 & 1 & 1 & 1 & 3 \\
15.5 & 0 & 0 & 0 & 0 \\
16.0 & 2 & 0 & 1 & 2 \\
16.5 & 1 & 1 & 2 & 4 \\
17.0 & 0 & 10 & 1 & 8 \\
17.5 & 2 & 14 & 1 & 12 \\
18.0 & 7 & 44 & 4 & 49 \\
18.5 & 23 & 46 & 6 & 60 \\
19.0 & 29 & 54 & 7 & 56 \\
\hline
\end{tabular}

well as results for 47 Tuc and 3 other fields in $\omega$ Cen will be published elsewhere.

\section{Observations and data reduction}

The OGLE project is conducted using the 1-m Swope telescope at Las Campanas Observatory which is operated by Carnegie Institution of Washington. A single $2048 \times 2048$ pixels Loral CCD chip, giving the scale of 0.435 arcsec/pixel is used as the detector. The initial processing of the raw frames is done automatically in near-real time. Details of the standard OGLE processing techniques are described by Udalski et al. (1992).

This paper is based in the data obtained during 1993 and 1994 observing seasons. Each season the cluster was monitored during about 3 months. Detailed logs of observations can be found in Udalski et al. (1994b, 1995). In 1993 we monitored three fields. Fields 5139A and 5139C were centered about 13 arcmin North and about 13 arcmin South of the cluster center, respectively. Field 5139B covered central part of the cluster. In 1994 we monitored mostly field 5139BC. This field is offset by about 0.5 arcmin South relatively to the field $5139 \mathrm{~B}$ - such a shift was applied in order to include in the central field a newly 
Table 7. Rectangular and equatorial coordinates for variables identified in $\omega$ Cen. The $X$ and $Y$ coordinates give positions of variables on the template images (see text). The last column gives alternative names for three variables which were previously known

\begin{tabular}{|c|c|c|c|c|c|c|}
\hline Name & $X$ & $Y$ & $\begin{array}{r}\mathrm{RA}(2000) \\
(\mathrm{h}: \mathrm{m}: \mathrm{s})\end{array}$ & $\begin{array}{r}\operatorname{Dec}(2000) \\
\left(0^{\prime}:^{\prime \prime}\right) \\
\end{array}$ & Field & $\begin{array}{l}\text { Other } \\
\text { Name }\end{array}$ \\
\hline OGLEGC1 & 458.02 & 693.52 & $13: 26: 20.45$ & $-47: 31: 59.77$ & $5139 \mathrm{BC}$ & \\
\hline OGLEGC2 & 796.44 & 794.47 & $13: 26: 34.55$ & $-47: 31: 03.44$ & $5139 \mathrm{BC}$ & \\
\hline OGLEGC3 & 687.99 & 1139.61 & $13: 26: 28.64$ & $-47: 28: 38.03$ & $5139 \mathrm{BC}$ & \\
\hline OGLEGC4 & 1606.97 & 1401.50 & $13: 27: 06.90$ & $-47: 26: 10.17$ & $5139 \mathrm{BC}$ & \\
\hline OGLEGC5 & 1829.46 & 1603.02 & $13: 27: 15.62$ & $-47: 24: 34.54$ & $5139 \mathrm{BC}$ & NJL79 \\
\hline OGLEGC6 & 353.63 & 1019.25 & $13: 26: 11.04$ & $-47: 15: 53.90$ & 5139A & \\
\hline OGLEGC7 & 1048.81 & 1524.93 & $13: 26: 38.85$ & $-47: 11: 51.45$ & 5139A & \\
\hline OGLEGC8 & 1967.81 & 966.84 & $13: 27: 19.91$ & $-47: 15: 21.08$ & 5139A & \\
\hline OGLEGC9 & 1450.04 & 1676.90 & $13: 27: 07.87$ & $-47: 37: 05.26$ & 5139C & \\
\hline OGLEGC10 & 862.94 & 1905.14 & $13: 26: 33.28$ & $-47: 23: 00.11$ & $5139 \mathrm{BC}$ & \\
\hline OGLEGC11 & 1485.72 & 42.80 & $13: 27: 06.86$ & $-47: 36: 03.07$ & $5139 \mathrm{BC}$ & \\
\hline OGLEGC12 & 1389.98 & 1819.19 & $13: 26: 56.07$ & $-47: 23: 17.58$ & $5139 \mathrm{BC}$ & \\
\hline OGLEGC13 & 1691.80 & 1401.10 & $13: 27: 10.52$ & $-47: 26: 07.14$ & $5139 \mathrm{BC}$ & \\
\hline OGLEGC14 & 486.71 & 1898.47 & $13: 26: 17.27$ & $-47: 23: 16.98$ & $5139 \mathrm{BC}$ & \\
\hline OGLEGC15 & 1033.92 & 89.44 & $13: 26: 47.34$ & $-47: 35: 59.91$ & $5139 \mathrm{BC}$ & \\
\hline OGLEGC16 & 1188.07 & 1840.97 & $13: 26: 47.38$ & $-47: 23: 15.73$ & $5139 \mathrm{BC}$ & \\
\hline OGLEGC17 & 1983.73 & 1732.20 & $13: 27: 21.71$ & $-47: 23: 32.78$ & $5139 \mathrm{BC}$ & \\
\hline OGLEGC18 & 1948.51 & 1967.27 & $13: 27: 19.31$ & $-47: 21: 52.40$ & $5139 \mathrm{BC}$ & NJL5 \\
\hline OGLEGC19 & 1640.67 & 1897.75 & $13: 27: 02.67$ & $-47: 08: 49.10$ & 5139A & \\
\hline OGLEGC20 & 1769.64 & 1618.77 & $13: 27: 21.76$ & $-47: 37: 19.14$ & $5139 \mathrm{C}$ & \\
\hline OGLEGC21 & 524.39 & 1107.09 & $13: 26: 30.08$ & $-47: 41: 44.32$ & $5139 \mathrm{C}$ & \\
\hline OGLEGC22 & 191.66 & 917.77 & $13: 26: 08.24$ & $-47: 30: 32.52$ & $5139 \mathrm{BC}$ & \\
\hline OGLEGC23 & 319.91 & 1630.78 & $13: 26: 12.64$ & $-47: 24: 42.14$ & 5139B & $\mathrm{V} 2$ \\
\hline OGLEGC24 & 390.20 & 1247.84 & $13: 26: 16.91$ & $-47: 27: 25.60$ & 5139B & \\
\hline OGLEGC25 & 335.06 & 743.76 & $13: 26: 11.21$ & $-47: 17: 53.44$ & $5139 \mathrm{~A}$ & \\
\hline OGLEGC26 & 253.62 & 541.02 & $13: 26: 08.44$ & $-47: 19: 23.55$ & 5139A & \\
\hline OGLEGC27 & 1165.27 & 150.63 & $13: 26: 48.75$ & $-47: 21: 40.79$ & 5139A & \\
\hline OGLEGC28 & 1801.04 & 84.63 & $13: 27: 16.14$ & $-47: 21: 47.28$ & $5139 \mathrm{~A}$ & \\
\hline OGLEGC29 & 260.73 & 1200.98 & $13: 26: 18.45$ & $-47: 41: 12.57$ & $5139 \mathrm{C}$ & \\
\hline OGLEGC30 & 1309.84 & 599.78 & $13: 26: 53.25$ & $-47: 18: 22.43$ & $5139 \mathrm{~A}$ & \\
\hline OGLEGC31 & 613.12 & 539.02 & $13: 26: 23.77$ & $-47: 19: 12.35$ & $5139 \mathrm{~A}$ & \\
\hline OGLEGC32 & 1476.66 & 1551.36 & $13: 27: 02.27$ & $-47: 24: 36.63$ & 5139B & \\
\hline OGLEGC33 & 250.61 & 994.51 & $13: 26: 10.48$ & $-47: 29: 57.10$ & $5139 \mathrm{BC}$ & \\
\hline OGLEGC34 & 412.13 & 1819.46 & $13: 26: 14.37$ & $-47: 23: 53.94$ & $5139 \mathrm{BC}$ & \\
\hline OGLEGC35 & 902.04 & 409.20 & $13: 26: 40.50$ & $-47: 33: 46.35$ & $5139 \mathrm{BC}$ & \\
\hline OGLEGC36 & 1766.28 & 1691.67 & $13: 27: 12.59$ & $-47: 23: 58.56$ & $5139 \mathrm{BC}$ & \\
\hline OGLEGC37 & 361.07 & 435.27 & $13: 26: 25.29$ & $-47: 46: 40.93$ & $5139 \mathrm{C}$ & \\
\hline OGLEGC38 & 1721.53 & 1819.38 & $13: 27: 19.01$ & $-47: 35: 53.87$ & $5139 \mathrm{C}$ & \\
\hline OGLEGC39 & 543.09 & 1568.20 & $13: 26: 17.23$ & $-47: 11: 49.97$ & $5139 \mathrm{~A}$ & \\
\hline OGLEGC40 & 1146.90 & 314.63 & $13: 26: 47.35$ & $-47: 20: 30.86$ & $5139 \mathrm{~A}$ & \\
\hline
\end{tabular}

discovered detached eclipsing binary which was located in the overlapping area of fields 5139B and 5139C. Field $5139 \mathrm{C}$ was also monitored for a few nights at the beginning of the 1994 observing season. Fields 5139A and $5139 \mathrm{C}$ overlap by about 1.5 arcmin with fields 5139B and 5139BC. The equatorial coordinates of centers of four observed fields are given in Table 1.

Most of the monitoring was performed through the Johnson $V$ filter. Only a few exposures in the KronCousins $I$ band were obtained for fields monitored during the 1993 season. A substantial number of exposures in the $I$ band were obtained for fields $5139 \mathrm{C}$ and $5139 \mathrm{BC}$ during the 1994 season. Table 2 gives total numbers of frames reduced for each of surveyed fields. For fields 5139A, 5139BC and $5139 \mathrm{C}$ and for the $V$ filter the exposure times ranged from $420 \mathrm{sec}$ to $600 \mathrm{~s}$, with $420 \mathrm{~s}$ being the most frequent value. Most of the $V$ frames of field 5139B were taken with the exposure time set to 240 s. For majority of the analyzed frames seeing was better than 1.6 arcsec.

The DoPHOT photometry program (Schechter et al. 1993) was used to derive profile-fitting photometry. We used DoPHOT in the fixed-position mode. The stellar positions were provided from a list obtained by reduction of "template" images. Separate template list were used for $V$ and $I$ observations. For fields 5139A and 5139C individual frames of particularly good quality served as templates. In case of field $5139 \mathrm{BC}$ a template image for the $V$ filter was constructed by averaging 3 individual frames. Frames mr4534, mr6489-91 (average of three individual frames), mr4535 and mr4704 were used as templates for fields 5139A, 5139BC, 5139B and 5139C, respectively. All frames collected by the OGLE team were deposited at 
the NASA NSS Data Center ${ }^{1}$. To cope with positional

Table 8. Light-curve parameters for SX Phe stars from the field of $\omega$ Cen. $A_{V}$ is the range of observed variations in the $V$ band. $(V-I)$ is the observed color at the maximum light. The period is given in days

\begin{tabular}{rrrrrr}
\hline Name & Period & $\langle V\rangle$ & $V_{\max }$ & $A_{V}$ & $(V-I)$ \\
\hline OGLEGC1 & 0.04712 & 17.03 & 16.95 & 0.13 & 0.58 \\
OGLEGC2 & 0.04818 & 17.41 & 17.32 & 0.15 & 0.71 \\
OGLEGC3 & 0.06229 & 16.65 & 16.21 & 0.63 & 0.65 \\
OGLEGC4 & 0.04952 & 16.72 & 16.52 & 0.32 & 0.47 \\
OGLEGC5 & 0.06549 & 16.79 & 16.56 & 0.36 & 0.50 \\
OGLEGC6 & 0.05065 & 17.20 & 17.09 & 0.19 & 0.54 \\
OGLEGC7 & 0.04642 & 17.17 & 17.11 & 0.12 & 0.57 \\
OGLEGC8 & 0.04178 & 16.75 & 16.61 & 0.25 & 0.49 \\
OGLEGC9 & 0.049375 & 16.95 & 16.71 & 0.40 & 0.41 \\
OGLEGC24 & 0.05326 & 17.19 & 17.11 & 0.2 & - \\
OGLEGC25 & 0.04374 & 17.14 & 17.10 & 0.07 & 0.53 \\
OGLEGC26 & 0.03867 & 17.35 & 17.31 & 0.08 & 0.57 \\
OGLEGC27 & 0.05289 & 17.05 & 17.00 & 0.10 & 0.54 \\
OGLEGC28 & 0.03613 & 16.74 & 16.71 & 0.05 & 0.71 \\
OGLEGC29 & 0.03891 & 17.31 & 17.28 & 0.05 & 0.55 \\
OGLEGC32 & 0.04864 & 16.99 & 16.85 & 0.25 & \\
OGLEGC33 & 0.03785 & 17.43 & 17.39 & 0.08 & 0.57 \\
OGLEGC34 & 0.038 & 17.38 & 17.35 & 0.05 & 0.62 \\
OGLEGC35 & 0.03985 & 17.35 & 17.30 & 0.08 & 0.50 \\
OGLEGC36 & 0.03753 & 17.40 & 17.36 & 0.08 & 0.54 \\
OGLEGC37 & 0.03388 & 16.55 & 16.56 & 0.03 & 0.48 \\
OGLEGC38 & 0.03748 & 17.52 & 17.55 & 0.05 & 0.62 \\
OGLEGC39 & 0.03697 & 17.56 & 17.58 & 0.04 & 0.058 \\
OGLEGC40 & 0.03654 & 17.36 & 17.40 & 0.10 & 0.53 \\
\hline
\end{tabular}

changes of the point spread function each analyzed frame was divided into a $4 \times 4$ grid of overlapping sub-frames. The point spread function showed only very small variability in these $540 \times 540$ pixel sub-images. Photometry derived for the "template" sub-frames was transformed to the common instrumental system by application of additive corrections. These corrections were equal to the aperture corrections derived for each sub-frame. Subsequently an instrumental photometry derived for a given sub-frame of a given frame was tied to the common instrumental system of the "template" image. The CCD camera used by OGLE is known to suffer from some non-linearity (e.g. Udalski et al. 1993). This leads to systematic errors of photometry reaching up to $7 \%$ over the whole range of observed magnitudes for stars measured on individual frames. Therefore, a non-linear transformation was applied while transforming instrumental magnitudes to the systems defined by "template" images.

\footnotetext{
${ }^{1}$ The OGLE data (FITS images) are accessible for astronomical community from the NASA NSS Data Center. Send e-mail to: archives@nssdc.gfc.nasa.gov with the subject line: REQUEST OGLE ALL and put requested frame numbers (in the form MR00NNNN where NNNN stands for frame number according to OGLE notation), one per line, in the body of the message. Requested frames will be available using an "anonymous ftp" service from nssdc.gfc.nasa.gov host in location shown in the return e-mail message from archives@nssdc.gsfc.nasa.gov.
}

Finally the data base containing photometry from all reduced frames was constructed. Relatively poor measurements were flagged at this point. A given measurement was considered to be "poor" if the formal error of photometry was 2.5 times or more larger than the average error of photometry for the nearby stars of comparable magnitudes. The actual procedure of constructing the data base was similar to that described by Szymański \& Udalski (1993). Separate data bases were prepared for objects detected on the "template" images and for additional "nontemplate" objects measured by Dophot on the individual frames. Data bases for the $V$-band observations were constructed for all four monitored fields. Data bases for the $I$ band were constructed for fields 5139C and 5139BC, only. Only single frames in the $I$-band were reduced for fields 5139A and 5139B.

The data were calibrated using the following procedure. First we obtained VI photometry for the field located east of the cluster center (field 5139D in Udalski et al. 1995). This field includes several secondary standards calibrated by Walker (1994). Instrumental photometry for field 5139D was transformed to the standard system using relations:

$$
\begin{array}{r}
v=\text { const }+V-0.028 \times(V-I) \\
v-i=\text { const }+0.952 \times(V-I)
\end{array}
$$

where a lower case letters correspond to the instrumental magnitudes. The color terms of this transformation were determined using photometry for several Landolt (1993) fields. The zero points were derived using local standards from Walker (1994). Specifically, 9 stars with $16<V<18$ were used as secondary standards. This range of magnitudes was selected to obtain the possibly most reliable calibration for stars located near the turnoff on the cluster color-magnitude diagram. In Fig. 1 we plot the residuals between Walker's photometry and our calibrated data. Some systematic trends in residuals, which are due to nonlinearity of the camera used by OGLE, are apparent.

Subsequently stars from the overlapping part of fields 5139D and 5139BC were used to establish zero points of VI photometry for stars from field 5139BC. Finally stars from the overlapping parts of field $5139 \mathrm{BC}$ and three remaining fields were used to determine zero points of $V I$ photometry for these fields. The instrumental $v$ and $i$ magnitudes for stars included in data bases were transformed to the $V I$ system using simplified transformation in the form $V=v+$ const and $I=i+$ const. To determine value of offsets an average values of $(V-v)$ and $(I-i)$ were calculated for stars with $0.7<V-I<1.1$ The color term of the $V$ transformation equals 0.028 (see Eq. 1). Hence, neglection of this term leads to some systematic errors of $V$ magnitudes which do not exceed 0.028 mag for stars with $0<V-I<2.0$. Colors of variables stars discussed later in this paper range from $V-I \approx 0.3$ to $V-I \approx 1.1$. In fact the external errors of presented photometry are 
Table 9. Light-curve parameters for variables OGLEGC10-23 from the field of $\omega$ Cen. $(V-I)$ is the observed color at the maximum light. $T_{0}$ is the time of minimum light. Variable OGLEGC22 is listed twice - the first entry corresponds to the 1993 season while the second one corresponds to the 1994 season

\begin{tabular}{rllrrrr}
\hline Name & Type & $\begin{array}{l}\text { Period } \\
\text { (days) }\end{array}$ & $V_{\max }$ & $V_{\min }$ & $\begin{array}{r}T_{0} \text { HJD } \\
2449000+\end{array}$ & $(V-I)$ \\
\hline OGLEGC10 & EW & 0.368712 & 17.31 & 17.65 & 81.7776 & 0.69 \\
OGLEGC11 & EW & 0.307348 & 17.20 & 17.50 & 81.9880 & 0.52 \\
OGLEGC12 & EW & 0.275865 & 17.79 & 18.10 & 464.7160 & 0.56 \\
OGLEGC13 & EW & 0.305525 & 17.12 & 17.44 & 81.7978 & 0.44 \\
OGLEGC14 & EA & 0.834420 & 16.64 & 16.92 & 81.6456 & 0.31 \\
OGLEGC15 & EA & 1.496605 & 16.96 & 17.58 & 82.6188 & 1.08 \\
OGLEGC16 & EA & 0.576233 & 18.15 & 19.38 & 81.9842 & 0.82 \\
OGLEGC17 & EA & 2.466760 & 17.27 & 17.60 & 82.3608 & 0.89 \\
OGLEGC18 & EA & 1.376188 & 16.02 & 17.15 & 82.3725 & 0.44 \\
OGLEGC19 & EW & 0.39823 & 16.22 & 16.51 & 81.8333 & 0.38 \\
OGLEGC20 & EW & 0.341803 & 16.61 & 16.78 & 81.7196 & 0.44 \\
OGLEGC21 & EW & 0.249260 & 17.86 & 18.05 & 81.7409 & 0.66 \\
OGLEGC22 & RS? & 22 & 15.115 & 15.232 & 81.6 & - \\
OGLEGC22 & RS? & 22 & 15.075 & 15.182 & 464.5 & 1.11 \\
OGLEGC23 & M & 236 & - & 15.78 & - & - \\
OGLEGC30 & RS? & 23 & 14.56 & 14.90 & 91.0 & - \\
OGLEGC31 & RS? & 34 & 15.13 & 15.21 & 83.3 & 1.12 \\
\hline
\end{tabular}

dominated by uncertainties introduced by nonlinearity of the used detector.

Table 10. List of frames used for construction of CMDs shown in Fig. 12

\begin{tabular}{lrcr}
\hline Frame & $\begin{array}{r}T_{\exp } \\
\text { sec }\end{array}$ & Filter & $\begin{array}{r}\text { FWHM } \\
\text { arcsec }\end{array}$ \\
\hline mr6489 & 420 & $V$ & 0.92 \\
$\mathrm{mr} 6490$ & 420 & $V$ & 0.92 \\
$\mathrm{~m} 6491$ & 300 & $V$ & 0.92 \\
$\mathrm{mr} 6492$ & 60 & $V$ & 0.94 \\
$\mathrm{mr} 6425$ & 300 & $I$ & 1.15 \\
$\mathrm{mr} 6821$ & 15 & $I$ & 1.06 \\
$\mathrm{mr} 6822$ & 60 & $I$ & 1.08 \\
\hline
\end{tabular}

A search for variables was conducted using the data bases for the $V$ filter. Three methods were employed.

The first method makes use of a $\chi^{2}$ statistic. We dropped from the analysis the frames suffering from poor seeing (the limiting value was set to 1.65 arcsec for fields 5139A and 5139C, and to 1.5 arcsec for fields 5139B and 5139BC) and frames with the very high level of sky background. The actual number of frames retained for fields 5139A, 5139B, 5139BC and 5139C, was 144, 100, 204 and 190, respectively. Measurements flagged as "poor" were rejected during extraction of the light curve for a given object. For every star which was measured on at least 100 frames for a given data set, we calculated a $\chi^{2}$ statistic (for field 5139B the minimal number of frames was reduced to 80). To take into account a noise introduced by the procedure of transforming photometry from different frames to the common photometric system and to compensate for the residual effects of variable PSF, we raised formal errors of photometry returned by DoPHOT by 0.02 mag. Objects with $P\left(\chi^{2}\right)<10^{-4}$ (e.g. Press et al. 1986) were considered candidate variables and their light curves were tested for variability with periods in the range from 0.03 to 60 days. To determine the most probable periods we used an aov statistic (Schwarzenberg-Czerny 1989, 1991). This statistic allows - in particular - reliable determination of periods for variables with non-sinusoidal light curves (e.g. eclipsing variables of EA-type). Phased light curves of suspected variables were subsequently inspected visually and objects with regular light curves were selected. In Fig. 2 we present a plot of rms deviation versus the average $V$ magnitude for the light curves of stars from field 5139A examined for variability. The analogous plot for field $5139 \mathrm{C}$ looks very much the same and therefore it is not shown. For fields 5139A and 5139C the search for variable stars was conducted down to $V=19.25$. For fields 5139B and 5139BC the quality of photometry degrades significantly with decreasing distance from the clusters center. Stars from these two fields were divided in two subgroups. For stars located inside central area of size $1000 \times 1000$ pixel $^{2}$ the search for variables was conducted down to $V=18.25$ and $V=17.75$ for fields 5139B and 5139BC, respectively. The outer regions of fields 5139B and 5139BC were searched for variables down to $V=18.75$. In Figs. 3 and 4 we plot rms vs. $<V>$ for the inner and outer parts of field 5139BC, respectively. Tables 3-5 give some information about the numbers of stars analyzed for variability and numbers of stars which were selected as suspected variables. The total number of stars contained in data bases ranged from about $1.1 \mathrm{E} 5$ for fields $5139 \mathrm{~B}$ and $5139 \mathrm{BC}$ to about $3.5 \mathrm{E} 4$ for fields 5139A and 5139C. Most of these stars were faint 
objects with $V>19$ showing noisy and poorly sampled light curves. Out of about 5300 candidate variables with an average magnitude $V>14.75$ which were selected in all 4 fields with a $\chi^{2}$ test, only 26 could be classified with confidence as periodic variables. The time-domain light curves of candidate variables with the average magnitude $V<18.0$ were examined visually. No obvious variables, with the exception of previously selected periodic stars, were noted.

The $\chi^{2}$ statistic allows reliable detection of variables showing sine-like light curves. It is relatively less suitable for detection of detached eclipsing binaries whose light curves are flat for most of the time and show only relatively short episodes of decreased brightness. Therefore the light curves extracted from data bases were scanned with a filter designed to detect probable eclipsing events. A candidate eclipse event was defined as a set of three consecutive points on a light curve which fulfill condition:

$$
V+3 \times \sigma>M(V)+\delta V
$$

where $\sigma$ is an error of a given measurement and $M(V)$ is a median value of magnitude for a given stars. Parameter $\delta V$ corresponds to the minimal depth of a potential eclipse detectable by the filter. We used $\delta V=0.10$ for stars with $M(V)<18.0$ and $\delta V=0.15$ for fainter objects. Stars whose light curves exhibited 2 or more possible eclipse events were selected as candidate variables. Table 6 provides numbers of candidates selected in each of four analyzed fields. Comparison of data from Table 6 and Tables 3-5 shows that of the two used methods the first one based on a $\chi 2$ test - leads to significantly higher frequency of false alarms. All eclipsing binaries discovered using an $\chi 2$ test were detected also by filtering light curves with an "eclipse filter". No new variables were however discovered using the second method of search. Two of the low amplitude variables with sine-like light light curves were discovered only using the $\chi 2$ test. Almost all variables which were included in more than one data base were detected independently in all relevant data sets. The only exceptions were SX Phe variable OGLEGC2 which was missed in field 5139BC, and an eclipsing binary OGLEGC15 which was missed in field 5139B. The names of variables quoted here refer to the Tables presented in the next section.

Both methods described above select candidate variables based on presence of some unexpected scatter in the light curves of examined stars. The criteria used to select possible variables were chosen as a compromise between possibility of missing some low-amplitude variables and danger of picking up unreasonably large number of false variables. The monitored fields are very crowded and for some stars the estimated errors of photometry are likely to be underestimated. Therefore application of statistical tests like $\chi 2$ leads to detection of huge numbers of false variables. On the other hand one may expect presence of some low-amplitude and periodic variables in $\omega$ Cen (e.g.

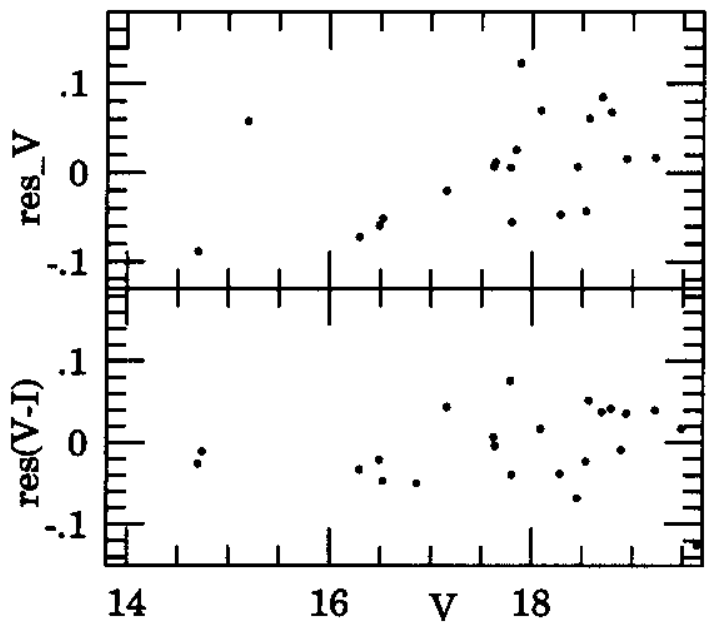

Fig. 1. Residuals for the Walker (1994) standards from field 5139D. The residuals are in the sense (Walker-OGLE)

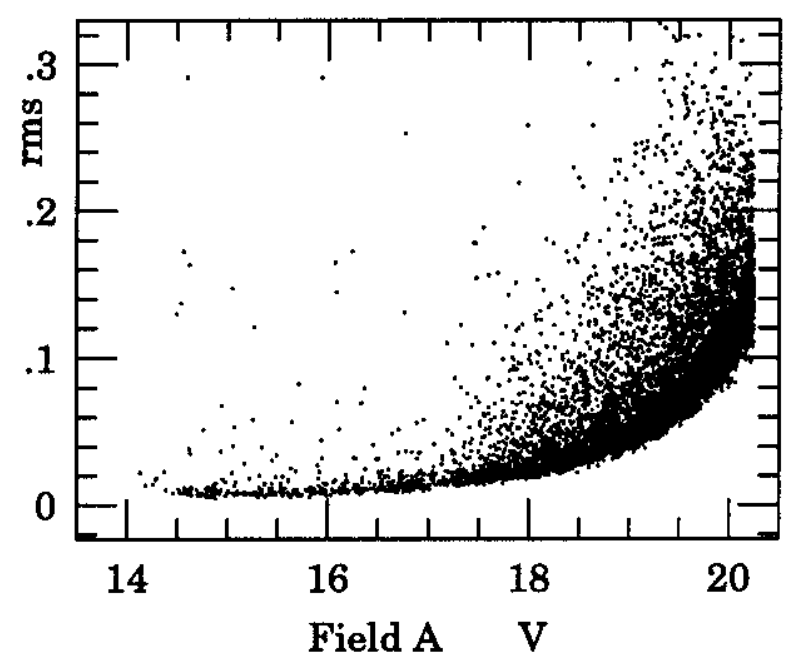

Fig. 2. Standard deviation versus average $V$ magnitude for stars with at least 100 reliable measurements. This plot corresponds to field 5139A. Only $1 / 3$ of the whole sample of stars examined for variability was plotted for clarity. Stars with $V>19.25$ are not shown as they were not examined for variability

SX Phe stars or contact binaries with low inclination). To look for this type of variables we calculated power spectra for all stars whose average magnitude was $V<18.1$. Only well populated light curves were subject to the analysis (the minimal number of "good" points qualifying a given light curve for analysis ranged from 90 for field 5139B to 150 for field $5139 \mathrm{BC}$ ). The power spectra were calculated using the CLEAN algorithm (Roberts et al. 1987). This particular algorithm is very efficient in removing from the derived power spectrum some false peaks introduced by periodicities which are present in the observing window. Stars whose power spectra showed a peak indicating 


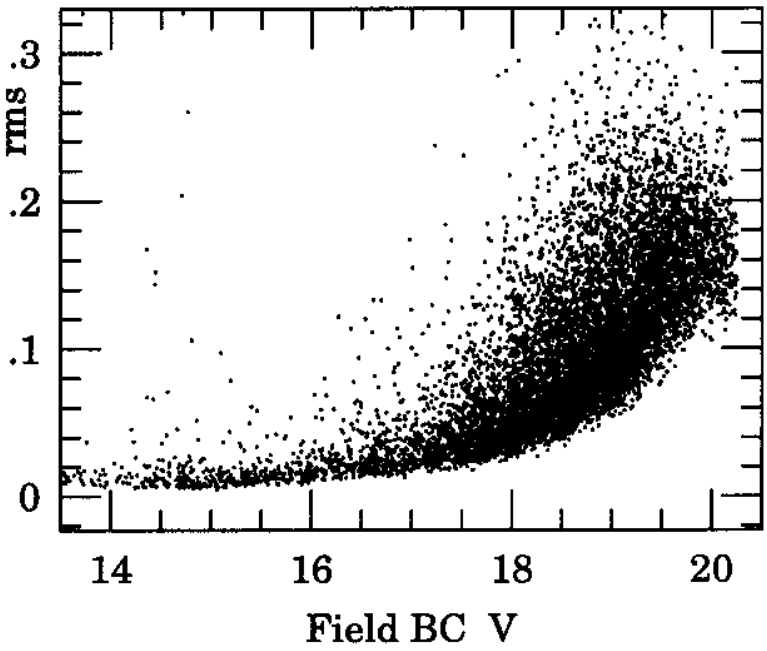

Fig. 3. Same as Fig. 1 but for the inner part of field 5139BC. Only $1 / 5$ of the whole sample was plotted for clarity

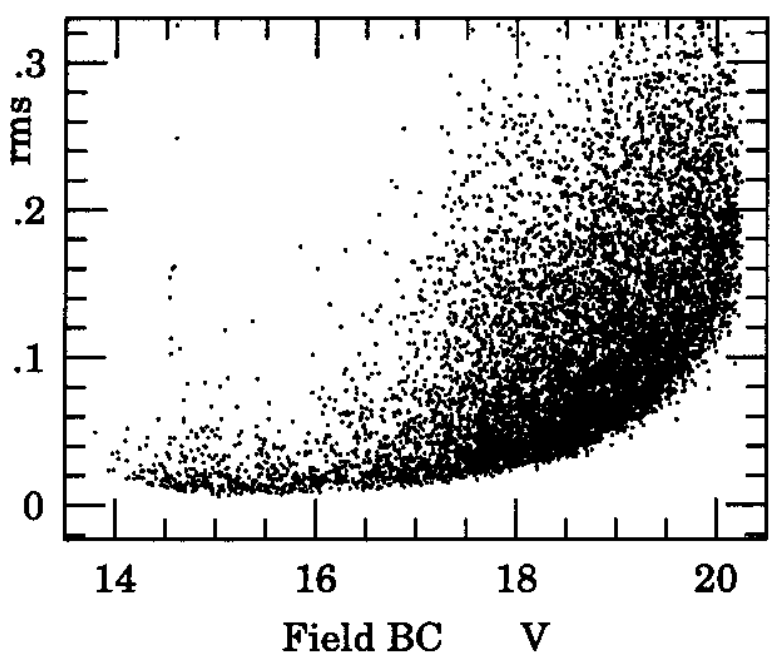

Fig. 4. Same as Fig. 1 but for the outer part of field 5139BC. Only $1 / 5$ of the whole sample was plotted for clarity

presence of modulation of the light curve with a full amplitude exceeding some specified threshold were selected as potential variables. The threshold was set at the level of $0.025 \mathrm{mag}$ for stars with $V<17.0$ and at the level of $0.04 \mathrm{mag}$ for stars with $17<V<18.1$. There were two reasons to limit this procedure to stars with $V<18.1$ : a) the light curves of fainter stars are too noisy to detect with confidence variables with amplitudes lower than about $0.1 \mathrm{mag}$; b) calculation of power spectra with CLEAN is quite demanding in respect of the CPU time. The number of selected candidates was about 60 for fields 5139A and raised to about 100 for fields 5139B and 5139BC. It should stated that this method of selecting variables works best for objects with smooth sine-like light curves. In particular, it works poorly for EA-type eclipsing bina- ries. The light curves of selected candidates were phased and examined visually. Sixteen stars proven to be real lowamplitude variables. These were variables OGLEGC2429, OGLEGC31-40. Their power spectra, with one exception, showed a single pronounced peak. Of these 16 variables 15 are SX Phe stars and one is a long period variable (OGLEGC31). Admittedly we did not use any formal statistical test to prove reality of detected variability of these low-amplitude variables. However, as we show below, 22 out of 24 detected SX Phe stars proven to be blue stragglers although blue stragglers constituted only a small fraction of all stars examined for variability. For two SX Phe stars position on the color-magnitude diagram is unknown as we are unable to determine their colors. It is unexpected to find SX Phe on the subgiant branch or among main-sequence stars. The fact that no one of identified SX Phe stars turned out to be located on the CMD outside area occupied by blue stragglers supports reality of detected variability. Several systems were observed during both observing seasons. For these stars very similar values of periods were obtained from two independent data sets.

Concluding this section we would like to emphasize that our goal was simply to detect the maximum number of variables of different types. The question on completeness of obtained sample of variables is beyond scope of this paper. An extensive tests would be necessary to answer the question about number of variables which were missed. A lower limits on the relative frequency of occurrence of some particular types of variables can be derived using data from Tables 3-6 and the data presented in the next section.

\section{Basic properties of variables}

The rectangular and equatorial coordinates of 40 periodic variables identified in our data are listed in Table 7 . The rectangular coordinates correspond to positions of variables on the $V$-band "template" images which were submitted to the editors of A\&A (see Appendix A). These images allow easy identification of all objects listed in Table 7 . The name of field in which a given variable can be identified is given in the 6th column.

For fields 5139A and 5139C transformation from rectangular to equatorial coordinates was derived based on positions of a set of stars from the Guide Star Catalogue (Lasker et al. 1988). We identified 101 and 95 GSC stars for fields 5139A and 5139C, respectively. The adopted plate solutions reproduce equatorial coordinates of GSC stars with residuals rarely exceeding 0.5 arcsec. Transformations for fields 5139BC and 5139B was derived based on positions of 69 RR Lyr variables. Equatorial coordinates for RR Lyr stars in $\omega$ Cen were kindly provided by Dr. Nicolai Samus (Shokin, Evstigneeva and Samus, in preparation). A few GSC stars were identified in the outer parts of fields 5139B and 5139BC. The adopted plate 
solution reproduces equatorial coordinates of these stars with residuals not exceeding 0.8 arcsec.

Our sample of variables consist of 24 SX Phe stars (variables 1-9, 24-29 and 32-40), 12 binaries (variables 10-21), three objects of uncertain classification (variables 22, 30 and 31) and one Mira (variable 23). Variability of OGLEGC5 and OGLEGC18 was first discovered by Niss et al. (1978) and these stars are known as NJL79 and NJL5, respectively. Photometric studies of NJL5 and NJL79 were published by Jensen \& Jorgensen (1985) and by Jorgensen \& Hansen (1984), respectively. OGLEGC23 is a previously known Mira-type variable listed as $V 2$ in Hogg (1973) catalogue. This star was near its minimum light during the 1993 observing season It was sufficiently faint at that time to be measured on most of 4 minute long exposures obtained for field 5139B. On the contrary $V 2$ was too bright during the 1994 observing season to be measured on images of field 5139BC (note also longer exposure times for the field $5139 \mathrm{BC}$ as compared with the field 5139B).

Table 8 lists basic characteristic of the light curves of 20 SX Phe star identified in our survey. The mean $V$ magnitudes were calculated by numerically integrating the phased light curves after converting them into intensity scale. The periods of all but one SX Phe stars are firmly established. The power spectra of the light curves calculated using CLEAN algorithm (Roberts et al. 1987) show prominent peaks at the positions corresponding to periods listed in Table 8. The only exception is OGLEGC34. Its power spectrum show several peaks which only slightly lower than the main peak occurring at $P \approx 0.038 \mathrm{~d}$. Our data for this low amplitude variable are too poor to prove or disprove its multi-periodic nature. OGLEGC34 was observed during 1993 and 1994 seasons. For both light curves the maximal peak in the power spectrum is observed at the period $P=0.038$ day. Photometric data for the remaining variables are given in Table 9. Phased light curves for stars 1-22 and 24-40 are shown in Figs. 5-7. Figure 8 shows the light curve of OGLEGC23 obtained during the 1993 season.

Figure 9 shows location of variables with known colors on the cluster color-magnitude diagram (CMD). For SX Phe stars marked positions correspond to the intensity-averaged magnitudes. For the remaining variables we marked positions corresponding to magnitude at maximum light. The main sequence and red giant branch are marked with double lines in Fig. 9. The large width of these sequences is due to the well known spread of metallicity exhibited by stars in $\omega$ Cen (Woolley et al. 1966). All SX Phe stars as well as all contact binaries and two non-contact binaries (OGLEGC14 and OGLEGC18) are located on the cluster CMD among candidate blue stragglers. An eclipsing binary OGLEGC16 is located right at the clusters turnoff while two detached bina- ries OGLEGC17 and OGLEGC15 occupy positions on the subgiant branch.

Light curves of OGLEGC14, OGLEGC15 and OGLEGC17 are flat outside eclipses what indicates that these binaries are detached systems. Each of these stars exhibits two minima of similar depth what implies similar effective temperatures of their components. By combining radial velocity curves with photometry one would be able to determine absolute parameters for components of OGLEGC14, OGLEGC15 and OGLEGC17. OGLEGC14 is a blue straggler and most probably its components exchanged mass in the past. Relatively long periods of OGLEGC15 and OGLEGC17 connected with their positions on the cluster CMD indicate that these systems evolved most probably without mass exchange between their components. Hence, determination of parameters for these two binaries can provide us with a direct measure of masses for the turnoff stars in $\omega$ Cen. Moreover, all three detached systems listed above can be used for determination of a distance to $\omega$ Cen. For eclipsing binaries the fractional accuracy of distance determination is equal to the fractional accuracy of the determination of radial velocity amplitudes, $K_{1}$ and $K_{2}$, as the distance is proportional to stellar diameters, which in turn are proportional to the $K$ values. Basing on a spectra collected with large telescopes and using a new "two-dimensional" cross-correlation technique TODOCOR (Zucker \& Mazeh 1994; Metcalfe et al. 1995) one may expect to determine radial velocity amplitudes for components of discussed detached binaries with an accuracy better than $1 \%$.

Variables OGLEGC22 and OGLEGC31 are located on the giant branch in the cluster CMD. The light curve of OGLEGC22 is unstable (see Fig. 6) what excludes its classification as an ellipsoidal variable. Most probably OGLEGC22 belongs to so called "spotted variables". It may be either an RS CVn-type star or an FK Com-type star. The same remark cane be made about a nature of variability observed for OGLEGC30 and OGLEGC31.

\subsection{Cluster membership of the variables}

The $\omega$ Cen cluster is located at an intermediate galactic latitude of $b=+15 \mathrm{deg}$. Therefore, one cannot assume a-priori that all variables listed in Table 7 are cluster members. Figure 10 shows the period versus absolute magnitude diagram for 20 SX Phe stars identified in our survey. The standard relations for the F-mode pulsators (solid line) and the H-mode pulsators (dashed line) and for $[\mathrm{Fe} / \mathrm{H}]=-2.2$ (upper line) and $[\mathrm{Fe} / \mathrm{H}]=-0.7$ (lower line) are also shown. The calibration $P-L-[\mathrm{Fe} / \mathrm{H}]$ was taken from Nemec et al. (1994). We adopted for the cluster an apparent distance modulus $(m-M)_{V}=13.86$ while calculating absolute magnitudes of SX Phe stars. The assumed range of metallicities is based on results published by Brown \& Wallerstein (1993) and Vanture et al. (1994). One can see that observed luminosities of SX Phe stars 


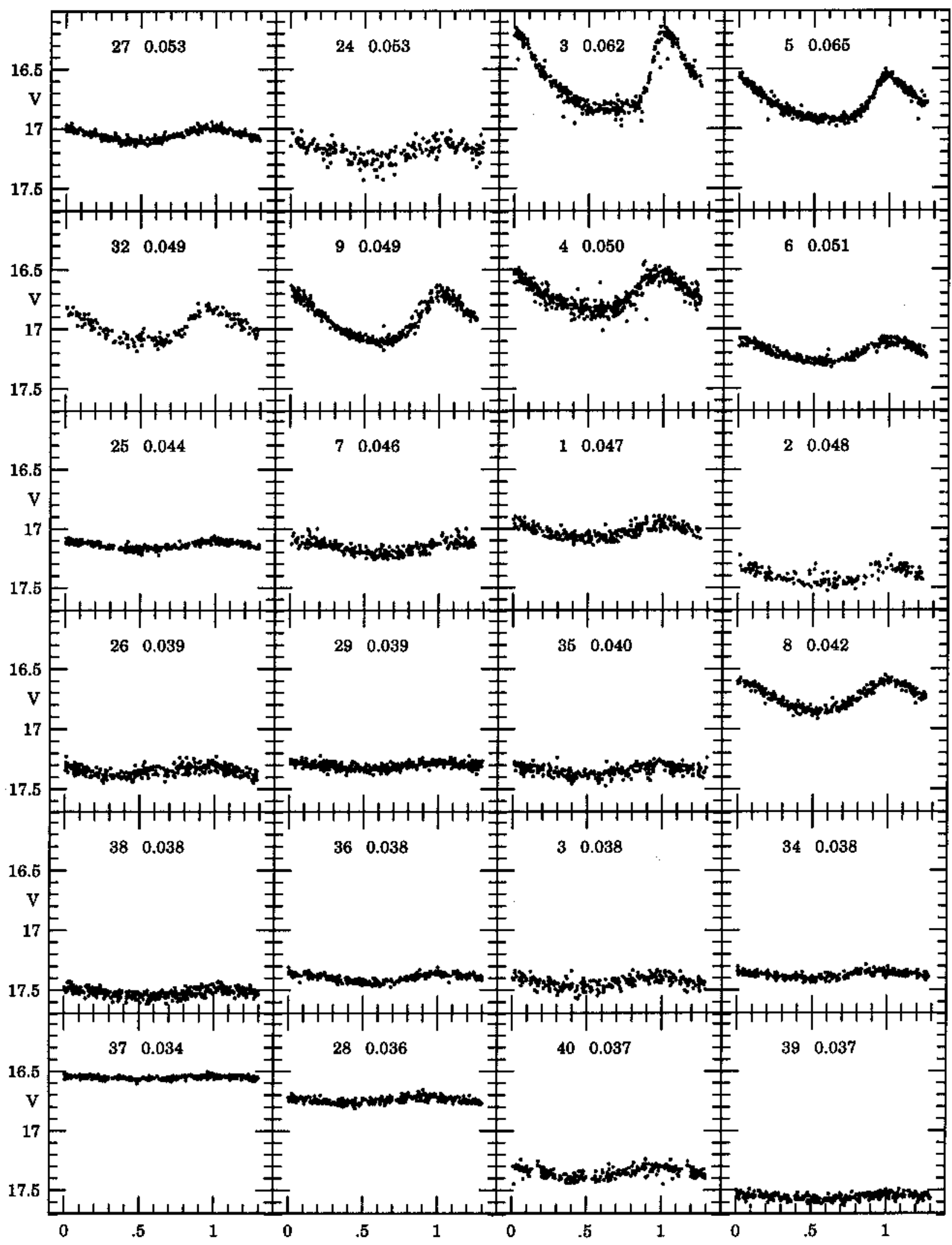

Fig. 5. Phased $V$ light curves for $24 \mathrm{SX}$ Phe stars identified in $\omega$ Cen. Inserted labels give numbers of variables and their periods. Note the same scale for all boxes. Variables are sorted according to their periods 

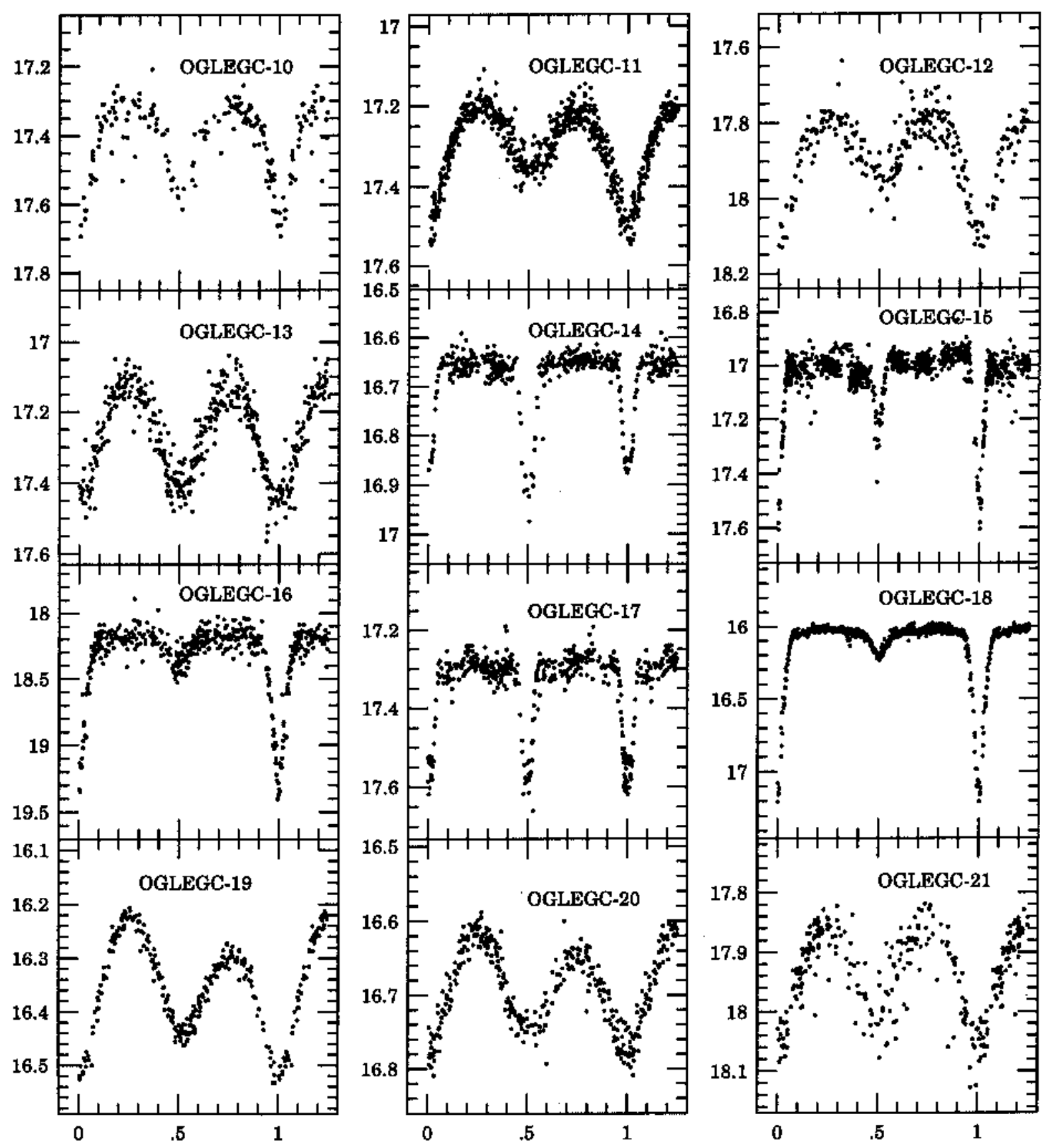

Fig. 6. Phased $V$ light curves for variables OGLEGC10-21

observed in the field of $\omega$ Cen are consistent with the hypothesis that all of them are members of the cluster.

We have applied the absolute brightness calibration established by Rucinski (1995) to calculate $M_{V}$ for newly discovered contact binaries. Rucinski's calibration gives $M_{V}$ as a function of period, unreddened color $(V-I)_{0}$ and metallicity:

$$
\begin{aligned}
M_{V}^{\text {cal }} & =-4.43 \log (P)+3.63(V-I)_{0}-0.31-0.12 \\
& \times[\mathrm{Fe} / \mathrm{H}] .
\end{aligned}
$$

We adopted for all systems $[\mathrm{Fe} / \mathrm{H}]=-1.6$ what is the mean metallicity for the cluster stars. Figure 11 shows the period versus an apparent distance moduli diagram for contact binaries identified in the surveyed area of $\omega$ Cen. An apparent distance modulus was calculated for each system as a difference between its $V$ magnitude and $M_{V}^{\text {cal }}$. An apparent distance modulus for $\omega$ Cen is estimated at $(m-M)_{V}=13.86$ (Nemec et al. 1994). We conclude based in Fig. 11 that the individual moduli calculated for each 


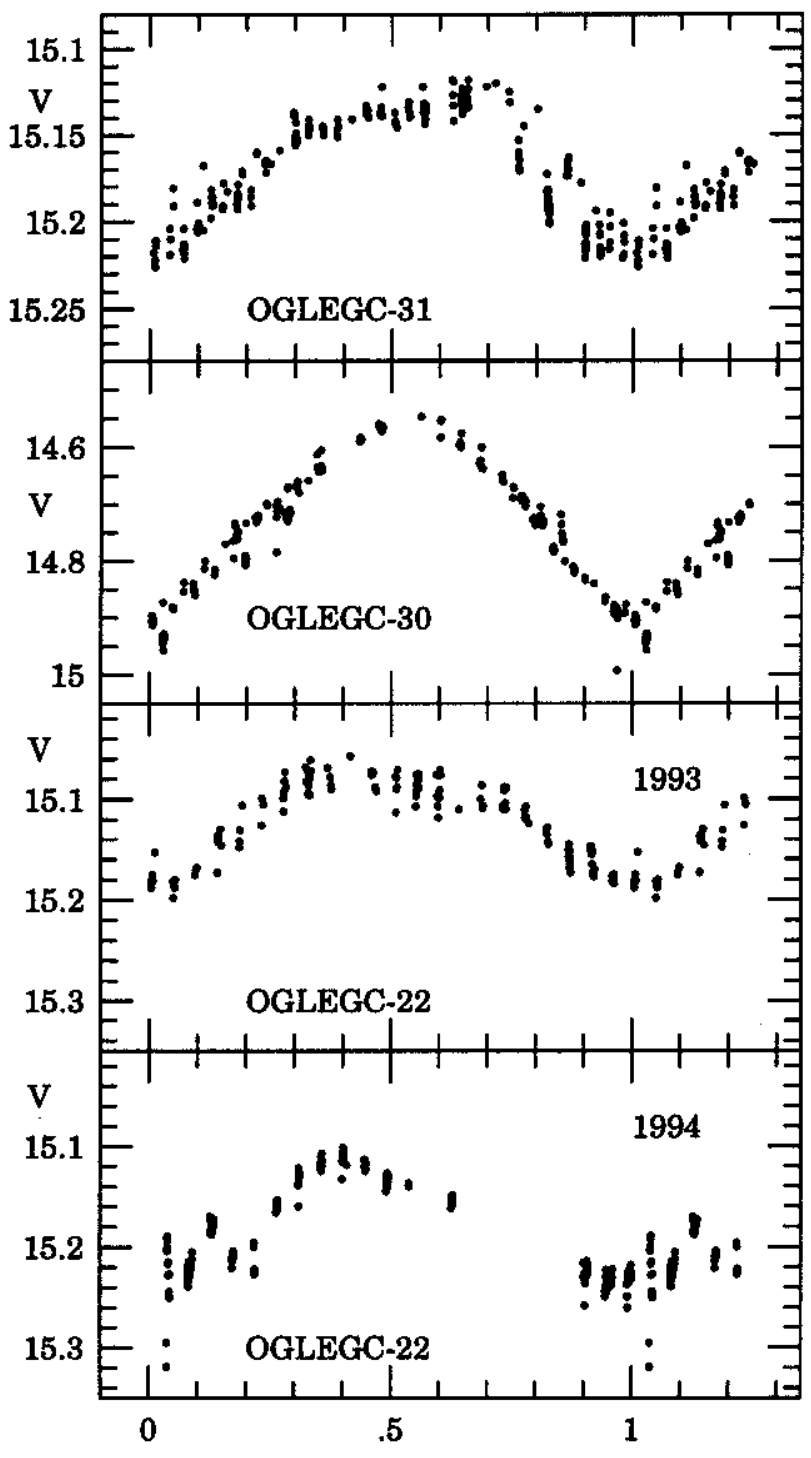

Fig. 7. Phased $V$ light curves for variables OGLEGC22, OGLEGC30 and OGLEGC31. Two light curves are shown for OGLEGC22. They correspond to 1993 (upper) and 1994 (lower) observing seasons

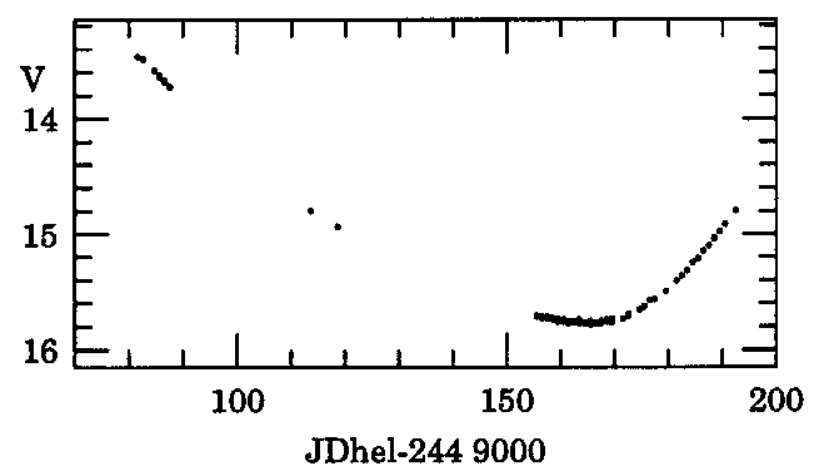

Fig. 8. The $V$-band light curve for variable OGLEGC23

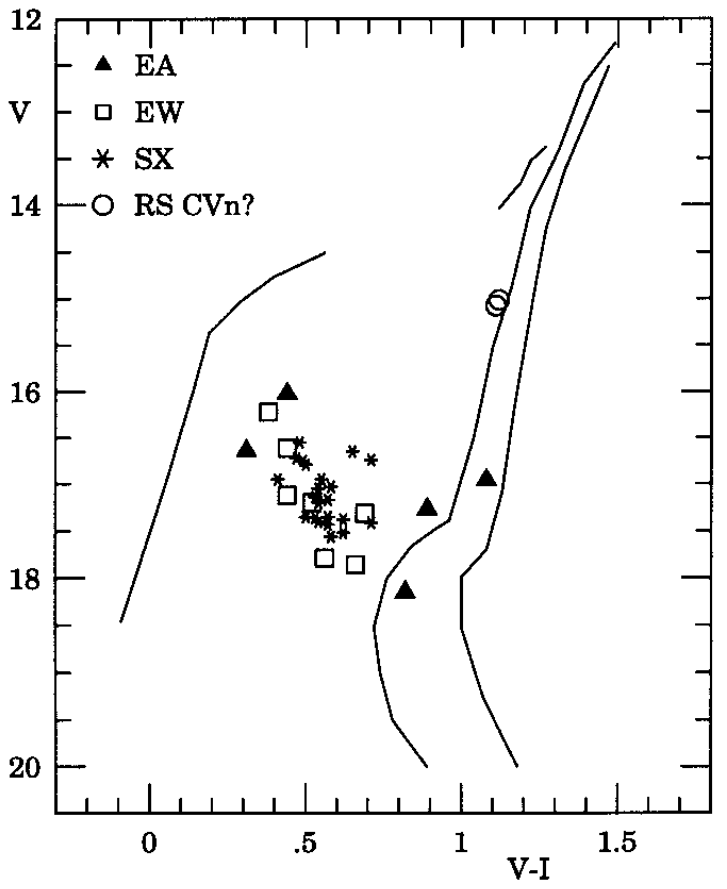

Fig. 9. The schematic CMD for $\omega$ Cen with positions of 37 variables marked. The squares represent contact binaries, the asterisks SX Phe stars and triangles non-contact eclipsing binaries. Probable RS CVn-type variables OGLEGC22 and OGLEGC31 are marked with open circles

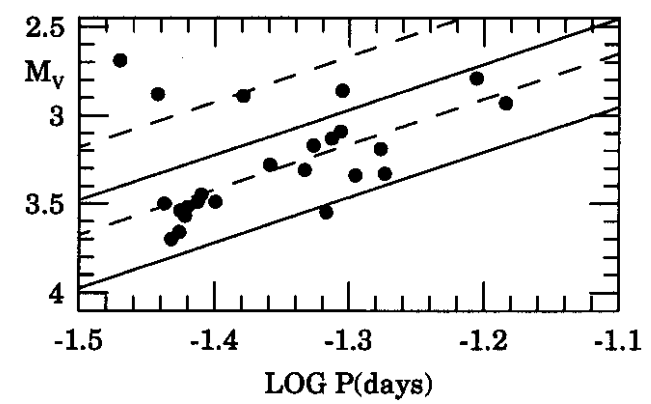

Fig. 10. Period, absolute magnitude diagram for SX Phe stars from the field of $\omega$ Cen

individual contact binary are compatible with their cluster membership.

\section{The color-magnitude diagrams}

In Fig. 12 we show the $V$ vs. $V-I$ CMDs of three surveyed fields. For each field the photometry is based on a single pair of $V \& I$ exposures - the $V$-filter "templates" (see 1'st paragraph of Sect. 4) were supplemented by the deepest available exposures in the $I$-band. We obtained $V I$ photometry for a more than 1E5 stars from all 3 fields. The presented CMDs were cleaned from stars of relatively poor photometry. It is known that $\omega$ Cen possesses an 


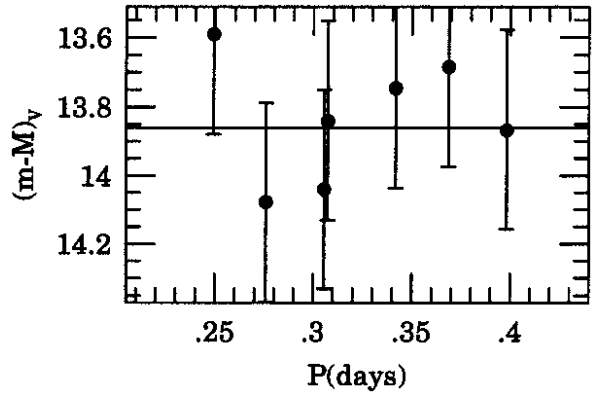

Fig. 11. Period, apparent distance modulus diagram for contact binaries from the field of $\omega$ Cen. A horizontal line at $(m-M)_{V}=13.86$ corresponds to distance modulus of the cluster. Error bars correspond to the formal uncertainty of absolute magnitudes derived using Rucinski's (1995) calibration

extended horizontal branch (Da Costa et al. 1986; Bailyn et al. 1992). Our data show that a sequence of blue stars extends down to $V \approx 19$. Moreover, the data for fields $5139 \mathrm{~A}$ and $5139 \mathrm{C}$ indicate that the sequence of faint blue stars has a gap approximately at $17.4<V<18.0$.

Another feature which can easily be noted on CMDs for fields $5139 \mathrm{~A}$ and $5139 \mathrm{C}$ is a scattered sequence of stars located to the blue of the subgiant branch of the cluster. The distribution of colors for these stars shows a cut-off at $(V-I) \approx 0.70$. This scattered sequence corresponds most probably to the foreground stars from the so called "old disk" (Jonch-Sorensen \& Knude 1994; Ng et al. 1995).

A substantial number of blue straggler candidates can be identified in CMD's for fields 5139A and 5139C. The accuracy of photometry for stars from field $5139 \mathrm{BC}$ is relatively low due increasing crowding as we move toward the cluster center. In Fig. 13 we show CMD for these stars from the field 5139BC whose photometry fulfills conditions $\sigma_{V} \leq 0.025$ and $\sigma_{B V} \leq 0.035$. Several candidates for blue stragglers are visible in this figure. In addition to photometry presented in Fig. 12 we constructed another CMDs for the field 5139BC. The CMD obtained by averaging photometry extracted from long and short exposures is show in Fig. 13a. Figure 13b presents CMD based on frames mr6492 and mr6822 while Fig. 13c shows CMD based on frames mr6492 and mr6821. Some information about frames used to construct CMDs presented in Fig. 13 are given in Table 10. Again presented CMDs were cleaned from stars of relatively poor photometry. An onset of the asymptotic giant branch at $V \approx 14.0$ can be noted in Figs. 12a-c.

All photometry presented in this section was submitted in tabular form to the editors of A\&A and is available in electronic form to all interested readers (see Appendix A). The potential users of these data should keep in mind a presence of some systematic magnitude dependent errors of the photometry (see Sect. 2).

\section{Summary}

An extended survey of the central part of $\omega$ Cen lead to discovery of 37 new periodic non-RR Lyr variables. We obtained also light curves of 3 previously known variables. Our sample of variables includes 24 SX Phe stars, 7 contact binaries, 5 non-contact eclipsing binaries and three likely spotted variables. All identified SX Phe stars and all contact binaries occupy positions among blue stragglers in the cluster CMD. Three non-contact binaries are located among turnoff stars. Of particular interest are detached systems OGLEGC15 and OGLEGC17 which can potentially provide information about masses of turnoff stars in $\omega$ Cen.

In the next paper from this series we shall present results for another $3 \omega$ Cen fields which were monitored by the OGLE team during the 1995 observing season. A separate paper will be devoted to RR Lyr stars in $\omega$ Cen.

Acknowledgements. This project was supported by NSF grants AST 92-16494 and AST-9313620 to Bohdan Paczynski and AST 92-16830 to George Preston. MK, MS and AU were supported by the Polish KBN grant 2P03D02908 to Andrzej Udalski. JK was supported by the Polish KBN grant 2P03D00808. JK wishes to thank Tomek Plewa for countless tips about Cshell scripts. We are indebted to Dr Nicolai Samus for providing us with equatorial coordinates of RR Lyr stars in $\omega$ Cen. We thank Dr Fusi Pecci for valuable suggestions included in his referee report.

\section{Appendix A}

Tables containing light curves of all variables discussed in this paper as well as tables with $V I$ photometry for more then 1E5 stars from the surveyed fields are published by A\&A at the centre de Données de Strasbourg, where they are available in electronic form: See the Editorial in A\&A 1993, Vol. 280, page E1. Equatorial coordinates are given for all stars included in these tables. We have also submitted the $V$-filter "template" images of four analyzed fields (see Sect. 2). These images can be used for identification of all variables discussed in this paper as well as for identification of all stars for which we provide $V I$ photometry.

\section{References}

Bailyn C.D., Sarajedini A., Cohn H., Luqqyer P.M., Grindlay J.E., 1992, AJ 103, 1325

Brown J.A., Wallerstein G., 1993, AJ 106, 133

Da Costa G.S., Norris J., Villumsen J.V., 1986, ApJ 308, 743

Gilliland R.L., Edmonds P.D., Petro L., Saha A., Shara M.M., 1995, ApJ 447, 191

Hogg H.S., 1973, Publ. DDO 6, No. 3, p. 1

Hodder P.J.C., Nemec J.M., Richer H.B., Falhman G.G., 1992, AJ 103, 460

Hut P., et al., 1992, PASP 104, 981

Jensen K.S., Jorgensen H.E., 1985, A\&AS 60, 229

Jonch-Sorensen H., Knude J., 1994, A\&A 288, 139

Jorgensen H., Hansen L., 1984, A\&A 133, 165

Kaluzny J., Krzeminski W., 1993, MNRAS 264, 785 
Landolt A.U., 1992, AJ 104, 340

Lasker B.M., et al., 1988, ApJS 68, 1

Mateo M.M., Harris H., Nemec J., Olszewski E., 1990, AJ 100, 496

Metcalfe T.S, Mathieu R.D., Latham D.W., Torres G., 1995, ApJ 456, 356

Nemec J.M., Linnell Nemec A.F., Lutz T.E., 1994, AJ 108, 222

Ng Y.K., Bertelli G., Bressan A., Chiosi C., Lub J., 1995, A\&A 295,66

Niss B., Jorgensen H.E., Lautsen S., 1978, A\&AS 32, 387

Paczyński B., et al., 1995, IAU Symp. 169: Unsolved Problems of the Milky Way. In: Blitz L. (ed.)

Press W.H., Flannery B.P., Teukolsky S.A., Verrerling W.T., 1986, Numerical Recipes, The Art of Scientific Computing. Cambridge Univ. Press, p. 165

Roberts D.H., Lehar J., Dreher J.W., 1987, AJ 93, 968

Rucinski S.M., 1995, PAPS 107, 648

Schechter P., Mateo M., Saha A., 1993, PASP 105, 1342

Schwarzenberg-Czerny A., 1989, MNRAS 241, 153
Schwarzenberg-Czerny A., 1991, MNRAS 253, 198

Szymański M., Udalski A., 1993, Acta Astron. 43, 91

Udalski A., Szymański M., Kaluzny J., Kubiak M., Mateo M., 1992, Acta Astron. 42, 253

Udalski A., Szymański M., Kaluzny J., Kubiak M., Mateo M., 1993, Acta Astron. 43, 69

Udalski A., Szymański M., Kaluzny J., Kubiak M., Mateo M., Krzemiński W., 1994a, ApJ 426, L69

Udalski A., Szymański M., Kaluzny J., Kubiak M., Mateo M., Krzemiński W., 1994b, Acta Astron. 44, 1

Udalski A., Szymański M., Kaluzny J., Kubiak M., Mateo M., Krzemiński W., 1995, Acta Astron. 45, 237

Vanture A.D., Wallerstein G., Brown J.A., 1994, PASP 106, 835

Woolley R.v.dR., et al., 1966, R. Obs. Ann., No. 2

Walker A.R., 1994, PASP, 106828

Zucker S., Mazeh T., 1994, ApJ 420, 806

Yan L., Mateo M.M., 1994, AJ 108, 1810 


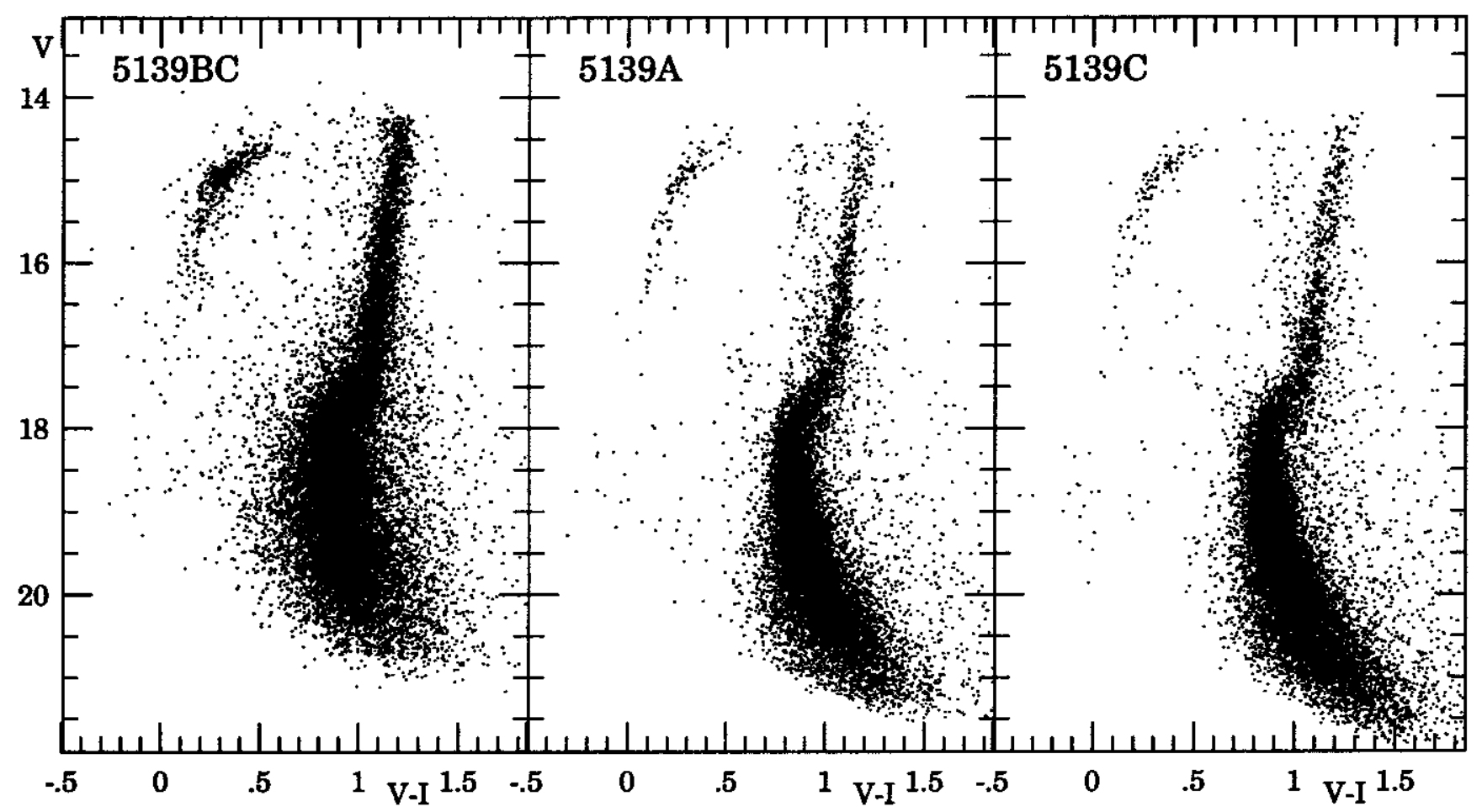

Fig. 12. The CMDs for fields 5139BC (left), 5139A (center) and 5139C (right)

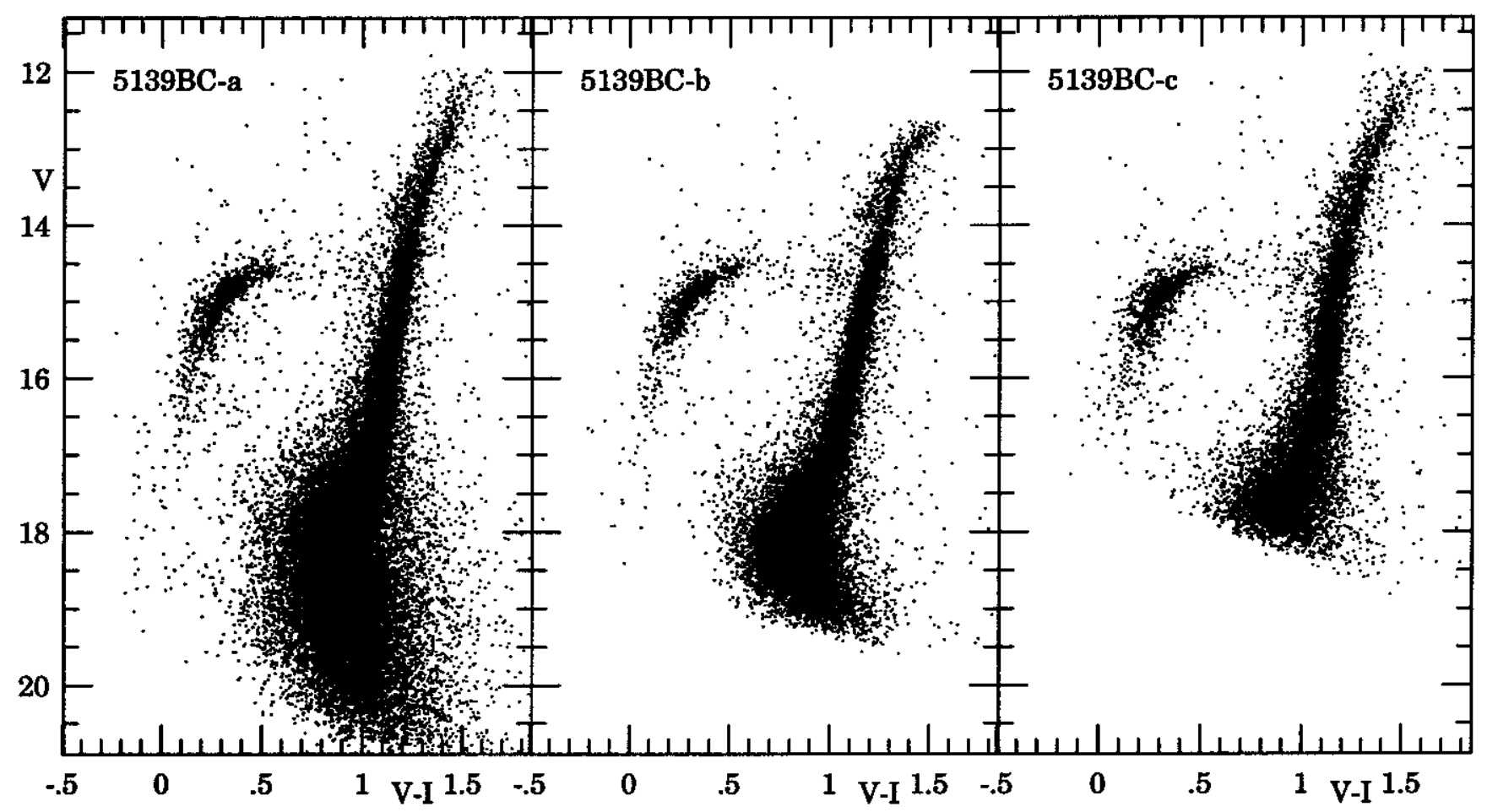

Fig. 13. The CMD's for field 5139BC (see text for details) 